\title{
Effect of Selective Head-Neck Cooling on Signs and Symptoms of Sport Originated Brain Injury in Amateur Sports: A Pilot Study
}

\author{
Doug King*1,2,3, Patria A Hume ${ }^{1}$, Karen Hind ${ }^{4}$ and Trevor Clark ${ }^{5}$ \\ ${ }^{1}$ Auckland University of Technology, New Zealand \\ ${ }^{2}$ University of New England, Australia \\ ${ }^{3}$ Massey University, New Zealand \\ ${ }^{4}$ Durham University, United Kingdom
}

${ }^{5}$ Australian College of Physical Education, Australia

*Corresponding author: Doug King, Department of Emergency, Hutt Valley District Health Board, Private Bag 31-07, Lower Hutt, New Zealand

\begin{abstract}
ARTICLE INFO
Received: 䌜 September 20, 2019

Published: 幽 October 01, 2019

Citation: Doug King, Patria A Hum, Karen Hind4, Trevor Clark5 and Cloe Cummins. Effect of Selective Head-Neck Cooling on Signs and Symptoms of Sport Originated Brain Injury in Amateur Sports: A Pilot Study. Biomed J Sci \& Tech Res 21(4)2019. BJSTR. MS.ID.003639.
\end{abstract}

Keywords: Concussion; Selective HeadNeck Cooling; Concussion Symptom; Hypothermia

\section{ABSTRACT}

Background: During contact sports, body temperature can elevate to between $38.9^{\circ} \mathrm{C}$ and $40.0^{\circ} \mathrm{C}$ at the peak of activity, and the temperature increase may be more long-lasting in hot environments. Mild traumatic brain injury (mTBI) occurring during exercise-induced hyperthermia may result in a cumulatively worse brain injury.

Aim: To investigate the effect of a portable head-neck cooling cap on people with sport originated brain injury (SOBI) and to record changes in the signs and symptoms of SOBI after a period of cooling (i.e. intervention) compared to people with SOBI and no cooling (i.e. control).

Methods: Thirty-five players with an identified SOBI were offered use of a head-neck cooling cap. Following assessment with the Concussion Symptom Scale (CSS) 13 players selected to rest and 22 players selected to wear the cooling cap for up to 30 minutes following the SOBI. Post CSS assessments were made following the rest or cooling cap period.

Results: Differences between cooling and non-cooling, irrespective of sex were a lower post Global Severity Index (14.6 \pm 4.8 vs. $18.9 \pm 4.4 ; \chi 2(1)=0.3 ; p=0.6091 ; t(12)=3.3$; $\mathrm{p}=0.0063 ; \mathrm{d}=0.93)$, Positive Symptom Total $(1.4 \pm 1.0$ vs. $2.9 \pm 0.8 ; \chi 2(1)=0.1 ; \mathrm{p}=0.7041$; $\mathrm{t}(12)=3.8 ; \mathrm{p}=0.0026 ; \mathrm{d}=1.66)$, CSS $(29.6 \pm 21.7$ vs. $64.5 \pm 17.6 ; \chi 2(1)=0.2 ; \mathrm{p}=0.7032$; $\mathrm{t}(12)=3.7 ; \mathrm{p}=0.0026 ; \mathrm{d}=1.77)$, and Positive Symptom Distress Index $(1.9 \pm 0.9$ vs. 3.2 $\pm 0.6 ; \chi 2(1)=0.2 ; \mathrm{p}=0.6682 ; \mathrm{t}(12)=3.6 ; \mathrm{p}=0.0033 ; \mathrm{d}=1.70$ ) for the cooling participants.

Conclusion: Despite limited participants, this pilot study showed initial positive support for the use of cooling in the management of SOBI to reduce the total symptom score and severity of the CSS when compared with rest alone.

\section{Highlights}

a) Brain temperature is slightly higher but correlates well with body temperature.

b) Brain hypothermia, with its broad and pleiotropic effects, presents as the most potent neuroprotection technique available to date.

c) Short duration, active head-neck cooling immediately after a SOBI may aid in acute phase recovery.

d) SOBI players described an improvement in feelings of wellbeing when using the Cryohelmet, and that their head instantly felt calmer and cooler. 


\section{Introduction}

In a resting individual, brain temperature is slightly higher but correlates well with body temperature. Differences in the correlation largely depend on the summed effects of brain metabolism, cerebral blood flow (CBF), volume and blood temperature [1]. The human brain utilizes approximately $25 \%$ of the body's energy allowance given it is a metabolically demanding organ with intense heat production [2,3]. There is a high thermal sensitivity of the brain to fluctuations in temperature exemplified by changes in brain temperature of $1^{\circ} \mathrm{C}$ or less resulting in nervous system functional alterations and energy deficiency [2]. During contact sports, body temperature can elevate to between $38.9^{\circ} \mathrm{C}$ and $40.0^{\circ} \mathrm{C}$ at the peak of activity, with the temperature increase possibly being longerlasting in hot environments [4-6]. The increased temperature can create a window of vulnerability where a cerebral injury can occur [1].

Hyperthermia during exercise is associated with hyperventilation that can result in a reduction in blood levels of carbon dioxide $[7,8]$. Carbon dioxide is a potent mediator of CBF, producing cerebral vasodilation, and this enhances cerebral heat clearance, narrowing the brain-blood temperature gradients [1] Conversely hyperventilation can induce hypocapnia, which causes cerebral vasoconstriction and widens the brain-blood gradients [9]. Cerebral carbon dioxide reactivity appears to increase during hyperthermia [10] and when combined with hyperventilationinduced hypocapnia, can lead to a notable reduction in CBF $[6,7]$. As a result, the temperature-associated reduction in carbon dioxide compromises cerebral heat clearance capacity when it is most needed physiologically [1]. As exercise increases cerebral metabolism and oxygen utilization, cerebral metabolic heat production concurrently increases [11-13]. The increase in cerebral metabolic heat production combined with the concomitant decrease in CBF leads to a meaningful cerebral heat storage [14].

This cerebral heat storage sustains a widened brain-body thermal gradient [1]. When exercise is terminated, the cerebral venous to arterial temperature difference increases, indicating a thermal recovery process from the net cerebral heat storage accumulated during exercise [14]. However, cerebral venous blood temperature decreases at a slower rate than body temperature, resulting in a relatively slow cerebral thermal recovery response from the exercise induced hyperthermia [14]. As a result, there is a temporal window of increased cerebral vulnerability to the deleterious effects from mild traumatic brain injury (mTBI) (i.e. formally known as concussion) both during and immediately after intense exercise [15]. Mild traumatic brain injuries instigate a neuronal, metabolic and cerebrovascular dysfunction, for example, CBF is reduced and glucose demands are elevated [16]. As such, mTBI occurring during exercise-induced hyperthermia, may result in a cumulatively worse head injury [17].

Experimental studies have shown that mild elevations in brain temperature $\left(<40^{\circ} \mathrm{C}\right)$ bring about worsening neurological outcomes [18] and there is a strong relationship between the incidence of fever and poor outcomes in patients with moderate to severe TBI [19]. However, there is a scarcity of studies reporting on brain temperature and the outcome of a mTBI. In a rodent model, mild brain temperature elevation before and after mTBI aggravated histopathological outcomes [5,15] and early posttrauma hyperthermia ( $<72 \mathrm{hr}$.) was closely correlated with unfavorable outcomes [20]. Despite these findings, the temporal window vulnerability may also present a window of opportunity for optimal selective brain temperature management. For example, there is a neuroprotective effect from the cooling of the head, largely attributed lowered temperatures decreasing the metabolic rate and reducing blood flow to the brain [21]. For every $1^{\circ} \mathrm{C}$ decrease in cerebral temperature there was a $5 \%$ decrease in glucose metabolism and brain oxygen compensation [22].

Laboratory studies have identified that brain hypothermia, with its broad and pleiotropic effects, presents as the most potent neuroprotection technique available to date $[1,23,24]$. The use of brain hypothermia has been well established in selected clinical conditions including hypoxic ischaemic neonatal encephalopathy [25] and anoxic brain injury due to cardiac arrest [26,27]. In the sporting realm, the use of a sideline cooling system is not a new concept, with some studies $[28,29]$ using a Spartan Cooling System (Welkins EMT Temperature Management System, Welkinsmed, Downers Grove, Il, USA). This system comprises a conditioning unit containing propylene glycol attached by an umbilical tubing to a headset facilitating cooling or heating by circulating the propylene glycol, enabling temperature ranges from $-20^{\circ} \mathrm{C}$ to $54^{\circ} \mathrm{C}$ [29]. One study [28] utilised this system to enhance aerobic performance in hot environments while another study [29] reported on the effect of cooling on physiological and cognitive functions in healthy volunteers. Other studies have utilised similar styles of head cooling with one study [24] reporting on intensive care unit patients requiring their heads to be shaved using the cooling cap for prolonged periods (48-72hr.) while another study [30] reported on a gel-based head-neck cooling device in healthy volunteers for 220 minutes.

Although studies [24,28-30] have shown different results for external head cooling, all have identified that non-invasive cooling of the brain was possible over a brief period. Exposure to cold stimulus can have a gradual increase in systolic and diastolic blood pressure [29,31,32]. However, not all [30] studies that used selective head-neck cooling have reported any negative side effects. To date most studies $[4,19,33]$ have only reported on changes in cognitive and physiological parameters of the participants. However, no study has reported on the effect of selective head-neck cooling to induce hypothermia and how this affects the signs and symptoms of mTBI. We were interested in sport originated brain injury (SOBI) [34] as a form of mTBI given the high incidence of these injuries $[35,36]$. Therefore, the purpose of this study was to investigate the 
effect of a portable head-neck cooling cap on people with SOBI and to record changes in the signs and symptoms of concussion after a period of cooling (i.e. intervention) compared to people with SOBI and no cooling (i.e. control).

\section{Methods}

This study was undertaken as a cohort study. Players from local domestic rugby union, rugby league, netball and football clubs were invited to participate in the research. A total of eight rugby union teams (four females; four male), two rugby league teams (one female; one male), three netball teams (female) and one football team (female) were enrolled in the study. The research procedures were approved by the Health and Disability Ethics Committee (19/ NTA/3) and the trial was registered with the Australian and New Zealand Clinical Trials Registry (ACTRN12619000017123) and the World Health Organisation (UTN: U1111-1225-8322). A total of 35 SOBI were recorded over the 2019 competition season for all the teams included in the study.

\section{Procedures}

Any player identified with a SOBI was immediately removed from further match participation and sat on the sideline. After the initial assessment by the individuals team medic, affected players were asked to complete the Concussion Symptom Scale (CSS) $[33,37]$. Players could self-select to be part of the cooling group $(n=22)$ or the control group $(n=13)$ at the recruitment stage of the study. Players in the control group were asked to complete the CSS and then sit on the sideline or in the changing room for 30 minutes. Following this period, the control group repeated the CSS, to identify if any changes in the signs and symptoms had occurred. Players who elected to be in the cooling group were asked to complete the CSS and were then provided with the Catalyst ${ }^{\mathrm{TM}}$ Cryohelmet to wear for 15 to 30 minutes. The time the Cryohelmet was worn was recorded. Following the cooling period, the player was then asked to complete the CSS again and scores were recorded as the pre- and post-cooling symptom assessments. All players identified as having a SOBI were referred for further medical evaluation and underwent supervised rehabilitation and return to activity in accordance with the respective sporting bodies guidelines.

\section{Concussion Symptom Scale}

The concussion symptom scale (CSS) is a validated symptom inventory for the assessment of concussion (i.e. SOBI) symptoms and one of the most commonly utilized concussion assessment tools [33,37]. Comprising 22 items and utilizing a 7-point Likert scale ranging from 0 (no symptom) to 6 (severe symptom), the CSS provides assessment of symptoms, along with a severity score. The symptoms are not specific to concussion given non-injured participants have recorded symptoms on the CSS [33]. Participants were asked to rate the extent to which they were currently experiencing each symptom. The CSS total symptom score was calculated by counting the number of symptoms (range 0-22). The symptom severity was calculated by summing the value for each symptom (possible range: 0 -132). The CSS has a sensitivity of 0.64 to 0.89 and specificity of 0.91 to 1.0 and internal consistency reliability (Cronbach's $\alpha$ ) of 0.88 to 0.94 [33,38,39].

The CSS was assessed by symptom clusters consisting of physical (7 items; range 0-42; Cronbach's $\alpha=0.89$ ), cognitive (5 items; range 0-30; Cronbach's $\alpha=0.89$ ), sleep (4 items; range 0-24; Cronbach's $\alpha=0.79$ ) and affective (4 items; range 0-24; Cronbach's $\alpha=0.78$ ) factors $[39,40]$. The CSS scores were then transformed into global indices of distress variables [41]. These were global severity index (GSI), positive symptom total (PST) and positive symptom distress index (PSDI). The GSI was calculated by dividing the Concussion Symptom Severity (CSS) total score by 22 (the total number of symptoms that could be endorsed). The PST was then calculated by counting the total number of positively endorsed symptoms (0-22). Whilst, the PSDI was calculated by dividing the CSS total symptom score by the PST value. As a result, the GSI serves as an overall summary measure providing an indication of the average level of symptomology; the PST serves as a measure of symptom breadth; and the PSDI serves as a measure of symptom intensity [42].

\section{Cooling Helmet}

Players were provided with the Catalyst ${ }^{\mathrm{TM}}$ Cryohelmet (www. cryohelmet.com) to wear on the head and neck for the cooling period between assessments (Figure 1). The Cryohelmet consists of an insulating neoprene shell that is latex free and has a drawstring attached to enable adjustment for a better thermal transfer and contain the cold packs on the inside of the helmet (Figure 1A). The Catalyst cold packs are contained in an Ultracool fabric for moisture wicking and easy drying and are easily replaceable (Figure 1B). The Cryohelmet was placed in a freezer the night before the match was to occur and removed before the match started and stored in a self-contained transportable cooler pack. When a participant was identified as having a SOBI by the sports medic, they were removed from the match, completed the CSS evaluation and then the Cryohelmet was placed on their head and the neck wraps were applied with the cooling packs for a minimum of 15 minutes to facilitate cooling of the head and neck. Participants self-selected the duration of wearing the Cryohelmet but were encouraged to wear this for as long as they felt it was comfortable.

\section{Statistical Analysis}

All data collected were entered into a Microsoft Excel spreadsheet and analyzed with SPSS (IBM Corp, Released 2017. IBM SPSS Statistics for Windows, Version 25.0. Armonk, NY: IBM Corp). Data are reported as means with $95 \%$ confidence intervals (CI) [43]. A t-test was utilized to assess for differences in the number of SOBI recorded by male, female and by sporting code. A generalized linear model was utilized to determine whether there were differences in the pre- and post-CSS scores for both the cooling and control cohorts utilizing the post-score and post-cooling scores as the dependent variables. An inter-cohort 
analysis was also undertaken utilizing the control pre- and postscores as the dependent variable. If differences were detected, a post-hoc two-tailed paired t-test was utilized to determine if any significant differences existed. A Bonferroni-type adjustment was applied to maintain the Type- 1 error probability at the 0.05 alpha level. Cohen's effect sizes (d) were utilized to calculate practically meaningful differences between the measures of global indices of distress variables and the CSS symptom clusters [41]. Effect sizes of $<0.19,0.20-0.60,0.61-1.20$ and $>1.20$ were considered trivial, small, moderate, and large, respectively [44]. Risk-ratios were calculated for any differences identified between males and females and preand post-cooling on the CSS.

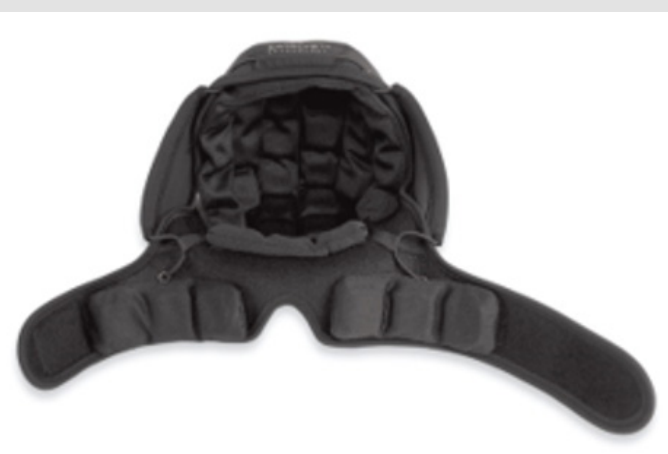

(A)

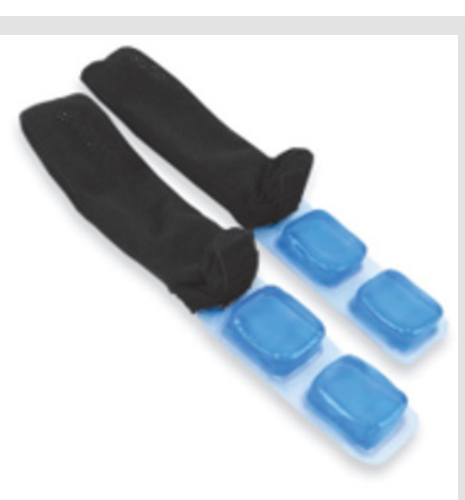

(B)

Figure 1: Catalyst Cryohelmet used for selective head-neck cooling showing position of cooling inserts (A) and reusable cold packs (B). [Pictures used with permission by Catalyst].

\section{Results}

A total of 35 SOBI were recorded across the teams that participated in the study (Table 1).

\section{Male Versus Female Differences}

There were more females enrolled in the study and they were older ( $29.2 \pm 7.9$ yr. vs. $24.5 \pm 4.7$ yr.; t (5) $=2.3$; $\mathrm{p}=0.0711$ ) than the males. Rugby league females $(31.7 \pm 2.3 \mathrm{yr}$.) were older than the rugby union $(29.8 \pm 4.9 ; \mathrm{t}(2)=1.1 ; \mathrm{p}=0.3942)$ and netball $(28.6$ $\pm 12.3 ; \mathrm{t}(4)=0.4 ; \mathrm{p}=0.7364)$ females. The mean GSI recorded for all concussion cases pre-cooling was $18.2 \pm 3.6$ (Table 2). Males recorded a higher pre-cooling GSI (18.5 \pm 2.1 vs. $18.1 \pm 4.1 ; \chi 2(1)$ $=3.9 ; \mathrm{p}=0.0492 ; \mathrm{t}(5)=-0.2 ; \mathrm{p}=0.8738 ; \mathrm{d}=0.12)$ than females. Females recorded a higher pre-cooling PST $(2.8 \pm 1.1$ vs. $2.5 \pm 0.8$; $\chi 2(1)=1.0 ; \mathrm{p}=0.3294 ; \mathrm{t}(5)=0.9 ; \mathrm{p}=0.4211 ; \mathrm{d}=0.31$ ) and had a higher pre-cooling CSS (60.4 \pm 24.8 vs. $54.0 \pm 17.3 ; \chi 2(1)=0.9 ; \mathrm{p}=0.3345$; $\mathrm{t}(5)=0.9 ; \mathrm{p}=0.4211 ; \mathrm{d}=0.30)$ and pre-cooling PSDI $(3.2 \pm 0.9$ vs. $2.9 \pm 0.7 ; \chi 2(1)=0.3 ; p=0.5576 ; t(5)=1.4 ; p=0.2998 ; d=0.37$ ) when compared with males.

Table 1: Summary of participants ages for selective head-neck cooling, no head-neck cooling and total participants by sporting code, gender, age and range of ages. All data reported as means \pm standard deviations.

\begin{tabular}{|c|c|c|c|c|c|c|c|c|c|}
\hline \multicolumn{4}{|c|}{ Female } & \multicolumn{3}{|c|}{ Male } & \multicolumn{3}{|c|}{ Total } \\
\hline Sporting Code & $\mathrm{n}=$ & Mean \pm SD & Range & $\mathrm{n}=$ & Mean \pm SD & Range & $\mathrm{n}=$ & Mean \pm SD & Range \\
\hline \multicolumn{10}{|c|}{ Selective head-neck cooling } \\
\hline Rugby Union & 8 & $28.8 \pm 4.1$ & 21 to 35 & 6 & $24.8 \pm 5.6$ & 18 to 35 & 14 & $26.9 \pm 5.5$ & 18 to 35 \\
\hline Rugby League & 3 & $31.7 \pm 2.3$ & 29 to 33 & 0 & - & - & 3 & $31.7 \pm 2.3$ & 29 to 33 \\
\hline Netball & 5 & $25.0 \pm 14.5$ & 18 to 51 & 0 & - & - & 5 & $25.0 \pm 14.5$ & 18 to 51 \\
\hline Total & 16 & $28.1 \pm 8.4$ & 18 to 51 & 6 & $24.8 \pm 5.6$ & 18 to 35 & 22 & $27.2 \pm 7.8$ & 18 to 51 \\
\hline \multicolumn{10}{|c|}{ No head-neck cooling } \\
\hline Rugby Union & 0 & - & - & 3 & $29.3 \pm 8.1$ & 22 to 38 & 3 & $29.3 \pm 8.1$ & 22 to 38 \\
\hline Rugby League & 0 & - & - & 3 & $23.7 \pm 4.5$ & 19 to 28 & 3 & $23.7 \pm 4.5$ & 19 to 28 \\
\hline Netball & 3 & $36.7 \pm 4.6$ & 32 to 40 & 0 & - & - & 3 & $34.7 \pm 4.6$ & 32 to 40 \\
\hline Football & 4 & $27.3 \pm 6.9$ & 19 to 36 & 0 & - & - & 4 & $27.3 \pm 7.0$ & 19 to 36 \\
\hline Total & 7 & $31.4 \pm 6.9$ & 19 to 40 & 6 & $26.5 \pm 6.6$ & 19 to 28 & 13 & $28.6 \pm 6.8$ & 19 to 40 \\
\hline \multicolumn{10}{|c|}{ Total } \\
\hline Rugby Union & 8 & $28.8 \pm 4.1$ & 21 to 35 & 8 & $24.9 \pm 5.0$ & 18 to 35 & 17 & $27.5 \pm 5.4$ & 18 to 38 \\
\hline Rugby League & 3 & $31.7 \pm 2.3$ & 29 to 33 & 3 & $23.7 \pm 4.5$ & 19 to 28 & 6 & $27.7 \pm 5.4$ & 19 to 33 \\
\hline Netball & 8 & $28.6 \pm 12.3$ & 18 to 51 & 0 & - & - & 8 & $28.6 \pm 12.3$ & 18 to 51 \\
\hline
\end{tabular}




\begin{tabular}{|c|c|c|c|c|c|c|c|c|c|}
\hline Football & 4 & $27.3 \pm 6.9$ & 19 to 36 & 0 & - & - & 4 & $27.3 \pm 6.9$ & 19 to 36 \\
\hline Total & 24 & $29.2 \pm 7.9$ & 18 to 51 & 11 & $24.5 \pm 4.7$ & 18 to 35 & 35 & $27.7 \pm 7.3$ & 18 to 51 \\
\hline
\end{tabular}

$\mathrm{SD}=$ Standard Deviation

Table 2: Summary of Concussion Symptom Scores (CSS) of selective head-neck cooling and no head-neck cooling for athletes with sport originated brain injury (SOBI) for global indices of distress variables [41] of sporting codes involved by gender and total scores.

All data reported as means \pm standard deviations.

\begin{tabular}{|c|c|c|c|c|c|c|c|c|c|c|c|c|c|c|c|}
\hline \multirow[b]{2}{*}{ Gender } & \multirow[b]{2}{*}{$\begin{array}{c}\text { Total } \\
\mathbf{n}=\end{array}$} & \multirow[b]{2}{*}{ Activity } & \multicolumn{3}{|c|}{ GSI } & \multicolumn{3}{|c|}{ PST } & \multicolumn{3}{|c|}{ CSS } & \multicolumn{3}{|c|}{ PSDI } & \multirow{2}{*}{$\begin{array}{c}\text { Duration } \\
\text { minutes } \\
\text { Mean } \\
\pm \text { SD }\end{array}$} \\
\hline & & & $\begin{array}{l}\text { Pre } \\
\text { Mean } \\
\pm \text { SD }\end{array}$ & $\begin{array}{l}\text { Post } \\
\text { Mean } \\
\pm \text { SD }\end{array}$ & $\begin{array}{c}\text { Diff } \\
\text { Mean } \\
\pm \text { SD }\end{array}$ & $\begin{array}{l}\text { Pre } \\
\text { Mean } \\
\pm \text { SD }\end{array}$ & $\begin{array}{l}\text { Post } \\
\text { Mean } \\
\pm \text { SD }\end{array}$ & $\begin{array}{c}\text { Diff } \\
\text { Mean } \\
\pm S D\end{array}$ & $\begin{array}{l}\text { Pre } \\
\text { Mean } \\
\pm \text { SD }\end{array}$ & $\begin{array}{l}\text { Post } \\
\text { Mean } \\
\pm \text { SD }\end{array}$ & $\begin{array}{c}\text { Diff } \\
\text { Mean } \\
\pm \text { SD }\end{array}$ & $\begin{array}{l}\text { Pre } \\
\text { Mean } \\
\pm \text { SD }\end{array}$ & $\begin{array}{l}\text { Post } \\
\text { Mean } \\
\pm \text { SD }\end{array}$ & $\begin{array}{l}\text { Diff } \\
\text { Mean } \\
\pm \text { SD }\end{array}$ & \\
\hline \multicolumn{16}{|c|}{ Selective Head-Neck Cooling } \\
\hline \multirow[t]{4}{*}{ Female } & 8 & Netball & $\begin{array}{c}18.2 \\
\pm 3.9^{\mathrm{a}}\end{array}$ & $\begin{array}{c}14.8 \\
\pm 6.1^{\mathrm{b}}\end{array}$ & $\begin{array}{c}3.4 \\
\pm 3.8\end{array}$ & $\begin{array}{c}2.6 \\
\pm 1.4^{\mathrm{a}}\end{array}$ & $\begin{array}{c}1.4 \\
\pm 1.3^{\mathrm{b}}\end{array}$ & $\begin{array}{c}1.2 \\
\pm 0.4\end{array}$ & $\begin{array}{c}57.0 \\
\pm 29.7^{\mathrm{a}}\end{array}$ & $\begin{array}{c}31.6 \\
\pm 28.4^{\mathrm{b}}\end{array}$ & $\begin{array}{l}25.4 \\
\pm 9.3\end{array}$ & $\begin{array}{c}3.0 \\
\pm 1.1^{\mathrm{a}}\end{array}$ & $\begin{array}{c}1.9 \\
\pm 1.0^{\mathrm{b}}\end{array}$ & $\begin{array}{c}1.1 \\
\pm 0.5\end{array}$ & $21.0 \pm 5.5$ \\
\hline & 3 & $\begin{array}{l}\text { Rugby } \\
\text { Union }\end{array}$ & $\begin{array}{c}17.9 \\
\pm 4.4^{\mathrm{a}}\end{array}$ & $\begin{array}{c}14.4 \\
\pm 5.8^{\mathrm{b}}\end{array}$ & $\begin{array}{c}3.5 \\
\pm 3.9\end{array}$ & $\begin{array}{c}2.8 \\
\pm 1.1^{\mathrm{a}}\end{array}$ & $\begin{array}{c}1.6 \\
\pm 1.2^{\mathrm{b}}\end{array}$ & $\begin{array}{c}1.3 \\
\pm 0.5\end{array}$ & $\begin{array}{c}62.1 \\
\pm 24.6^{\mathrm{a}}\end{array}$ & $\begin{array}{c}34.3 \\
\pm 26.6^{b}\end{array}$ & $\begin{array}{c}27.9 \\
\pm 11.2\end{array}$ & $\begin{array}{c}3.4 \\
\pm 0.9^{\mathrm{a}}\end{array}$ & $\begin{array}{c}3.4 \\
\pm 1.1^{\mathrm{b}}\end{array}$ & $\begin{array}{c}1.2 \\
\pm 0.4\end{array}$ & $20.6 \pm 5.0$ \\
\hline & 5 & $\begin{array}{l}\text { Rugby } \\
\text { League }\end{array}$ & $\begin{array}{c}18.7 \\
\pm 4.9^{\mathrm{a}}\end{array}$ & $\begin{array}{c}14.7 \\
\pm 3.2^{\mathrm{b}}\end{array}$ & $\begin{array}{c}4.0 \\
\pm 1.7\end{array}$ & $\begin{array}{c}2.8 \\
\pm 1.2^{\mathrm{a}}\end{array}$ & $\begin{array}{c}1.0 \\
\pm 0.5^{\mathrm{b}}\end{array}$ & $\begin{array}{c}1.8 \\
\pm 0.7\end{array}$ & $\begin{array}{c}61.7 \\
\pm 26.0^{\mathrm{a}}\end{array}$ & $\begin{array}{c}22.3 \\
\pm 10.7^{\mathrm{b}}\end{array}$ & $\begin{array}{c}39.3 \\
\pm 15.3\end{array}$ & $\begin{array}{c}3.3 \\
\pm 0.7^{\mathrm{a}}\end{array}$ & $\begin{array}{c}1.5 \\
\pm 0.5^{\mathrm{b}}\end{array}$ & $\begin{array}{c}1.8 \\
\pm 0.3\end{array}$ & $26.7 \pm 5.8$ \\
\hline & 16 & Total & $\begin{array}{c}18.1 \\
\pm 4.1^{\text {a }} \\
\end{array}$ & $\begin{array}{c}14.6 \\
\pm 5.2^{\mathrm{b}} \\
\end{array}$ & $\begin{array}{c}3.6 \\
\pm 3.4 \\
\end{array}$ & $\begin{array}{c}2.8 \\
\pm 1.1^{\mathrm{a}} \\
\end{array}$ & $\begin{array}{c}1.4 \\
\pm 1.1 \\
\end{array}$ & $\begin{array}{c}1.3 \\
\pm 0.5 \\
\end{array}$ & $\begin{array}{c}60.4 \\
\pm 24.8^{\mathrm{a}} \\
\end{array}$ & $\begin{array}{c}31.2 \\
\pm 24.1^{\mathrm{b}} \\
\end{array}$ & $\begin{array}{c}29.3 \\
\pm 11.8 \\
\end{array}$ & $\begin{array}{c}3.2 \\
\pm 0.9^{\mathrm{a}} \\
\end{array}$ & $\begin{array}{c}2.0 \\
\pm 1.0^{\mathrm{b}} \\
\end{array}$ & $\begin{array}{c}2.9 \\
\pm 0.7 \\
\end{array}$ & $21.9 \pm 5.4$ \\
\hline Male & 6 & $\begin{array}{l}\text { Rugby } \\
\text { Union }\end{array}$ & $\begin{array}{l}18.5 \\
\pm 2.1 \\
\end{array}$ & $\begin{array}{l}14.7 \\
\pm 4.1 \\
\end{array}$ & $\begin{array}{c}3.8 \\
\pm 3.5 \\
\end{array}$ & $\begin{array}{c}2.5 \\
\pm 0.8^{\mathrm{ad}} \\
\end{array}$ & $\begin{array}{c}1.2 \\
\pm 0.6^{\mathrm{b}} \\
\end{array}$ & $\begin{array}{c}1.3 \\
\pm 0.4 \\
\end{array}$ & $\begin{array}{c}54.0 \\
\pm 17.3^{\text {ad }} \\
\end{array}$ & $\begin{array}{c}25.3 \\
\pm 14.1^{\mathrm{b}} \\
\end{array}$ & $\begin{array}{l}28.7 \\
\pm 9.5 \\
\end{array}$ & $\begin{array}{c}2.9 \\
\pm 0.7^{\text {ad }} \\
\end{array}$ & $\begin{array}{c}1.6 \\
\pm 0.5^{\mathrm{b}} \\
\end{array}$ & $\begin{array}{c}1.3 \\
\pm 0.3 \\
\end{array}$ & $22.5 \pm 6.1$ \\
\hline Total & 22 & & $\begin{array}{c}18.2 \\
\pm 3.6^{\mathrm{a}}\end{array}$ & $\begin{array}{l}14.6 \\
\pm 4.8\end{array}$ & $\begin{array}{c}3.6 \\
\pm 3.3\end{array}$ & $\begin{array}{c}2.7 \\
\pm 1.0^{\mathrm{a}}\end{array}$ & $\begin{array}{c}1.4 \\
\pm 1.0\end{array}$ & $\begin{array}{c}1.3 \\
\pm 0.5\end{array}$ & $\begin{array}{c}58.7 \\
\pm 22.8^{\mathrm{a}}\end{array}$ & $\begin{array}{c}29.6 \\
\pm 21.7\end{array}$ & $\begin{array}{c}29.1 \\
\pm 11.0\end{array}$ & $\begin{array}{c}3.1 \\
\pm 0.9^{a}\end{array}$ & $\begin{array}{c}1.9 \\
\pm 0.9^{\mathrm{b}}\end{array}$ & $\begin{array}{c}1.3 \\
\pm 0.4\end{array}$ & $22.1 \pm 5.5$ \\
\hline \multicolumn{16}{|c|}{ No Head-Neck Cooling } \\
\hline \multirow[t]{3}{*}{ Female } & 3 & Netball & $\begin{array}{l}19.7 \\
\pm 2.5 \\
\end{array}$ & $\begin{array}{l}20.3 \\
\pm 2.1 \\
\end{array}$ & $\begin{array}{l}-0.7 \\
\pm 0.6 \\
\end{array}$ & $\begin{array}{c}3.1 \\
\pm 1.3 \\
\end{array}$ & $\begin{array}{c}3.1 \\
\pm 1.1 \\
\end{array}$ & $\begin{array}{c}0.0 \\
\pm 0.2 \\
\end{array}$ & $\begin{array}{c}68.7 \\
\pm 27.8 \\
\end{array}$ & $\begin{array}{c}67.7 \\
\pm 24.8 \\
\end{array}$ & $\begin{array}{c}1.0 \\
\pm 4.4 \\
\end{array}$ & $\begin{array}{c}3.4 \\
\pm 1.2 \\
\end{array}$ & $\begin{array}{c}3.3 \\
\pm 1.0 \\
\end{array}$ & $\begin{array}{c}0.2 \\
\pm 0.3 \\
\end{array}$ & $27.7 \pm 2.5$ \\
\hline & 4 & Football & $\begin{array}{l}19.8 \\
\pm 2.6\end{array}$ & $\begin{array}{l}19.8 \\
\pm 2.6\end{array}$ & $\begin{array}{c}0.0 \\
\pm 0.0\end{array}$ & $\begin{array}{c}2.9 \\
\pm 0.8\end{array}$ & $\begin{array}{c}2.7 \\
\pm 0.9\end{array}$ & $\begin{array}{c}0.1 \\
\pm 0.2\end{array}$ & $\begin{array}{c}64.5 \\
\pm 18.2\end{array}$ & $\begin{array}{c}63.0 \\
\pm 19.7\end{array}$ & $\begin{array}{c}1.5 \\
\pm 4.7\end{array}$ & $\begin{array}{c}3.3 \\
\pm 0.8\end{array}$ & $\begin{array}{c}3.2 \\
\pm 0.8\end{array}$ & $\begin{array}{c}0.1 \\
\pm 0.3\end{array}$ & $21.3 \pm 3.0$ \\
\hline & 7 & Total & $\begin{array}{l}19.7 \\
\pm 2.4 \\
\end{array}$ & $\begin{array}{l}20.0 \\
\pm 2.2 \\
\end{array}$ & $\begin{array}{l}-0.3 \\
\pm 0.5 \\
\end{array}$ & $\begin{array}{c}3.0 \\
\pm 0.9 \\
\end{array}$ & $\begin{array}{c}3.0 \\
\pm 0.9 \\
\end{array}$ & $\begin{array}{c}0.1 \\
\pm 0.2 \\
\end{array}$ & $\begin{array}{c}66.3 \\
\pm 20.7 \\
\end{array}$ & $\begin{array}{c}65.0 \\
\pm 20.1 \\
\end{array}$ & $\begin{array}{c}1.3 \\
\pm 4.2\end{array}$ & $\begin{array}{c}3.3 \\
\pm 0.9 \\
\end{array}$ & $\begin{array}{c}3.2 \\
\pm 0.8 \\
\end{array}$ & $\begin{array}{c}0.1 \\
\pm 0.3 \\
\end{array}$ & $24.0 \pm 4.3$ \\
\hline \multirow[t]{3}{*}{ Male } & 3 & $\begin{array}{l}\text { Rugby } \\
\text { Union }\end{array}$ & $\begin{array}{l}20.0 \\
\pm 2.0\end{array}$ & $\begin{array}{l}18.0 \\
\pm 2.0\end{array}$ & $\begin{array}{c}2.0 \\
\pm 0.0\end{array}$ & $\begin{array}{c}3.2 \\
\pm 1.0\end{array}$ & $\begin{array}{c}2.9 \\
\pm 0.8\end{array}$ & $\begin{array}{c}0.2 \\
\pm 0.1\end{array}$ & $\begin{array}{c}69.3 \\
\pm 21.0\end{array}$ & $\begin{array}{c}64.3 \\
\pm 18.5\end{array}$ & $\begin{array}{c}5.0 \\
\pm 2.6\end{array}$ & $\begin{array}{c}3.4 \\
\pm 4.7 \\
\end{array}$ & $\begin{array}{c}3.5 \\
\pm 0.6\end{array}$ & $\begin{array}{l}-0.1 \\
\pm 0.1\end{array}$ & $21.7 \pm 2.9$ \\
\hline & 3 & $\begin{array}{l}\text { Rugby } \\
\text { League }\end{array}$ & $\begin{array}{l}19.7 \\
\pm 2.5 \\
\end{array}$ & $\begin{array}{l}19.3 \\
\pm 2.5 \\
\end{array}$ & $\begin{array}{c}0.3 \\
\pm 0.6 \\
\end{array}$ & $\begin{array}{c}2.6 \\
\pm 0.4 \\
\end{array}$ & $\begin{array}{c}2.7 \\
\pm 0.5 \\
\end{array}$ & $\begin{array}{l}-0.1 \\
\pm 0.1 \\
\end{array}$ & $\begin{array}{l}57.3 \\
\pm 9.3 \\
\end{array}$ & $\begin{array}{c}58.7 \\
\pm 10.4 \\
\end{array}$ & $\begin{array}{l}-1.3 \\
\pm 1.2 \\
\end{array}$ & $\begin{array}{c}2.9 \\
\pm 0.1 \\
\end{array}$ & $\begin{array}{c}3.0 \\
\pm 0.2 \\
\end{array}$ & $\begin{array}{l}-0.1 \\
\pm 0.1 \\
\end{array}$ & $27.7 \pm 2.5$ \\
\hline & 6 & Total & $\begin{array}{l}19.8 \\
\pm 2.0 \\
\end{array}$ & $\begin{array}{l}18.7 \\
\pm 2.2 \\
\end{array}$ & $\begin{array}{c}1.2 \\
\pm 1.0 \\
\end{array}$ & $\begin{array}{r}2.9 \\
\pm 0.7 \mathrm{c} \\
\end{array}$ & $\begin{array}{c}2.8 \\
\pm 0.6 \\
\end{array}$ & $\begin{array}{c}0.1 \\
\pm 0.2 \\
\end{array}$ & $\begin{array}{r}63.3 \\
\pm 15.9 \mathrm{c} \\
\end{array}$ & $\begin{array}{c}61.5 \\
\pm 13.8 \\
\end{array}$ & $\begin{array}{c}1.8 \\
\pm 3.9 \\
\end{array}$ & $\begin{array}{c}3.2 \\
\pm 0.5 \mathrm{c} \\
\end{array}$ & $\begin{array}{c}3.3 \\
\pm 0.5 \\
\end{array}$ & $\begin{array}{l}-0.1 \\
\pm 0.1 \\
\end{array}$ & $24.7 \pm 4.1$ \\
\hline Total & 13 & & $\begin{array}{l}19.8 \\
\pm 2.1\end{array}$ & $\begin{array}{l}19.4 \\
\pm 2.2\end{array}$ & $\begin{array}{c}0.4 \\
\pm 1.0\end{array}$ & $\begin{array}{c}3.0 \\
\pm 0.8\end{array}$ & $\begin{array}{c}2.9 \\
\pm 0.8\end{array}$ & $\begin{array}{c}0.1 \\
\pm 0.2\end{array}$ & $\begin{array}{c}64.9 \\
\pm 17.9\end{array}$ & $\begin{array}{c}63.4 \\
\pm 16.9\end{array}$ & $\begin{array}{c}1.5 \\
\pm 3.9\end{array}$ & $\begin{array}{c}3.3 \\
\pm 0.7\end{array}$ & $\begin{array}{c}3.2 \\
\pm 0.6\end{array}$ & $\begin{array}{c}0.0 \\
\pm 0.2\end{array}$ & $24.3 \pm 4.0$ \\
\hline
\end{tabular}

GSI = Global Severity Index; PST = Positive Symptom Index; CSS = Concussion Symptom Score; PSDI = Positive Symptom Distress Index; ${ }^{*}=$ cooling period in minutes; Significant difference $(p<0.05)$ than $(a)=$ Post-cooling; $(b)$ Pre-cooling; $(c)=$ Non-Cooling

On average, males wore the cooling helmet for longer periods $(22.5 \pm 6.1 \mathrm{~min}$ vs. $21.9 \pm 5.4 \mathrm{~min} ; \chi 2(1)=0.9 ; \mathrm{p}=0.3468 ; \mathrm{t}(5)=-0.2$; $\mathrm{p}=0.8560 ; \mathrm{d}=0.10$ ) than females. There were notable differences in the pre- and post-cooling results for females on the GSI $(18.1 \pm 4.1$ vs. $14.6 \pm 5.2 ; \chi 2(1)=21.6 ; \mathrm{p}<0.0001 ; \mathrm{t}(15)=4.2 ; \mathrm{p}=0.0008 ; \mathrm{d}=0.75)$, PST $(2.8 \pm 1.1$ vs. $1.4 \pm 1.1 ; \chi 2(1)=57.2 ; \mathrm{p}<0.0001 ; \mathrm{t}(15)=9.9$; $\mathrm{p}<0.0001 ; \mathrm{d}=1.27), \operatorname{CSS}(60.4 \pm 24.8$ vs. $31.2 \pm 24.1 ; \chi 2(1)=57.2$; $\mathrm{p}<0.0001 ; \mathrm{t}(15)=9.9 ; \mathrm{p}<0.0001 ; \mathrm{d}=1.19)$ and PSDI $(3.2 \pm 0.9$ vs. $2.0 \pm 1.0 ; \chi 2(1)=54.4 ; \mathrm{p}<0.0001 ; \mathrm{t}(15)=11.3 ; \mathrm{p}<0.0001 ; \mathrm{d}=1.26$ ) over the duration of the study. There were no differences between GSI at pre-cooling (18.1 \pm 4.1 vs. $18.5 \pm 2.1 ; \chi 2(1)=0.9 ; \mathrm{p}=0.3324$; $\mathrm{t}(5)=0.1 ; \mathrm{p}=0.9360 ; \mathrm{d}=0.12)$ and post-cooling (14.6 \pm 5.2 vs. 14.7 $\pm 4.1 ; \chi 2(1)=0.0 ; \mathrm{p}=0.9104 ; \mathrm{t}(5)=0.4 ; \mathrm{p}=0.7055 ; \mathrm{d}=0.02)$ when comparing males and females. The differences between CSS at pre- cooling ( $60.4 \pm 24.8$ vs. $54.0 \pm 17.3 ; \chi 2(1)=1.3 ; p=0.2630 ; t(5)=0.6$; $\mathrm{p}=0.5717 ; \mathrm{d}=0.30)$ and post-cooling $(31.2 \pm 24.1$ vs. $25.3 \pm 14.1$; $\chi 2(1)=0.3 ; p=0.5579 ; t(5)=1.1 ; p=0.3089 ; d=0.30$ ) when comparing males and females were not significant. Interestingly both males and females $(29.3 \pm 11.8$ vs. $\pm 28.7 \pm 9.5 ; \chi 2(1)=0.4 ; p=0.5370 ; t(5)$ $=-0.9 ; \mathrm{p}=0.4036 ; \mathrm{d}=0.06$ ) had similar differences in CSS scores postcooling.

\section{Cooling Versus No Cooling Differences}

There was a two-fold difference in post-cooling scores when compared with pre-cooling scores for the GSI (RR: 1.7 [95\% CI: 1.4 to 2.1]; $\mathrm{p}<0.0001 ; \mathrm{d}=0.85$ ), PST (RR: 2.5 [95\% CI: 2.0 to 3.1]; $\mathrm{p}<0.0001$; $\mathrm{d}=1.30$ ), CSS (RR: 2.5 [95\% CI: 2.0 to 3.1]; $\mathrm{p}<0.0001$; $\mathrm{d}=1.31$ ) and PSDI (RR: 2.4 [95\% CI: 2.0 to 2.9]; $\mathrm{p}<0.0001$; $\mathrm{d}=1.33$ ) for all players. 
For the non-cooling group, there were no notable differences in pre and post rest scores on the CSS when examined by individual sport code and by gender. When compared by cooling and non-cooling, the cooling group recorded a lower post GSI $(14.6 \pm 4.8$ vs. $18.9 \pm 4.4$ $\chi 2(1)=0.3 ; \mathrm{p}=0.6091 ; \mathrm{t}(12)=3.3 ; \mathrm{p}=0.0063 ; \mathrm{d}=0.93)$, PST $(1.4 \pm 1.0$ vs. $2.9 \pm 0.8 ; \chi 2(1)=0.1 ; \mathrm{p}=0.7041 ; \mathrm{t}(12)=3.8 ; \mathrm{p}=0.0026 ; \mathrm{d}=1.66)$, CSS $(29.6 \pm 21.7$ vs. $64.5 \pm 17.6 ; \chi 2(1)=0.2 ; \mathrm{p}=0.7032 ; \mathrm{t}(12)=3.7$; $\mathrm{p}=0.0026 ; \mathrm{d}=1.77)$, and PSDI $(1.9 \pm 0.9$ vs. $3.2 \pm 0.6 ; \chi 2(1)=0.2$; $\mathrm{p}=0.6682 ; \mathrm{t}(12)=3.6 ; \mathrm{p}=0.0033 ; \mathrm{d}=1.70$ ), when compared with the non-cooling cohort. Furthermore, the non-cooling participants had a longer resting period before reassessment $(24.9 \pm 4.4 \mathrm{~min}$ vs 22.0 \pm 5.5 min; $\chi 2$ (1) =0.4; p=0.5188; $\mathrm{t}(12)=-2.5 ; \mathrm{p}=0.0281 ; \mathrm{d}=0.58$ ).
Females had a higher pre-cooling score for the physical cluster $(7.9 \pm 1.6$ vs. $5.5 \pm 2.4 ; \chi 2(1)=12.9 ; \mathrm{p}=0.0003 ; \mathrm{t}(14)=4.3 ; \mathrm{p}=0.0008$; $\mathrm{d}=0.27)$ and a higher pre-cooling severity (23.7 \pm 9.7 vs. 12.4 $\pm 10.1 ; \chi 2(1)=41.8$; pV0.0001; $\mathrm{t}(14)=8.4 ; \mathrm{p}<0.0001 ; \mathrm{d}=1.14$ ) when comparted with males (Table 3 ). There was a decrease in the score of the cognitive cluster of $0.7 \pm 0.8$ for the total selective headneck cooling group when comparing pre- $(4.7 \pm 0.7$ vs. $4.0 \pm 1.1$; $\chi 2(1)=16.5 ; \mathrm{p}<0.0001 ; \mathrm{t}(21)=4.1 ; \mathrm{p}=0.0005 ; \mathrm{d}=0.76)$ and post cooling. As a result, there was a decrease for the total selective head-neck cooling group in the symptom severity of $10.3 \pm 4.5$ when comparing pre- $(20.5 \pm 8.8$ vs. $8.8 \pm 6.1 ; \chi 2(1)=57.4 ; \mathrm{p}<0.0001$; $\mathrm{t}(21)=11.2 ; \mathrm{p}<0.0001 ; \mathrm{d}=1.55)$ and post-cooling.

Table 3: Summary of the Concussion Symptom Scale (CSS) clusters [39, 40] scores and severity for selective head-neck cooling and no head-neck cooling by gender and total. All data reported as means \pm standard deviations.

\begin{tabular}{|c|c|c|c|c|c|c|c|c|c|c|c|c|c|}
\hline \multicolumn{5}{|c|}{ Physical Factor } & \multicolumn{3}{|c|}{ Cognitive Factor } & \multicolumn{3}{|c|}{ Sleep Factor } & \multicolumn{3}{|c|}{ Affective Factor } \\
\hline \multicolumn{3}{|c|}{ Pre-Mean \pm SD } & $\begin{array}{l}\text { Post } \\
\text { Mean } \\
\pm \text { SD }\end{array}$ & $\begin{array}{c}\text { Diff } \\
\text { Mean } \\
\pm S D\end{array}$ & $\begin{array}{c}\text { Pre } \\
\text { Mean } \\
\pm \text { SD }\end{array}$ & $\begin{array}{l}\text { Post } \\
\text { Mean } \\
\pm \text { SD }\end{array}$ & $\begin{array}{l}\text { Diff } \\
\text { Mean } \\
\pm \text { SD }\end{array}$ & $\begin{array}{c}\text { Pre } \\
\text { Mean } \\
\pm \text { SD }\end{array}$ & $\begin{array}{c}\text { Post } \\
\text { Mean } \\
\pm \text { SD }\end{array}$ & $\begin{array}{c}\text { Diff } \\
\text { Mean } \\
\pm \text { SD }\end{array}$ & $\begin{array}{c}\text { Pre } \\
\text { Mean } \\
\pm \text { SD }\end{array}$ & $\begin{array}{c}\text { Post } \\
\text { Mean } \\
\pm \text { SD }\end{array}$ & $\begin{array}{c}\text { Diff } \\
\text { Mean } \\
\pm S D\end{array}$ \\
\hline \multicolumn{14}{|c|}{ Selective Head-Neck Cooling } \\
\hline \multirow[t]{2}{*}{ Female } & $\begin{array}{l}\text { Mean } \\
\text { Score }\end{array}$ & $7.5 \pm 1.6^{\mathrm{a}}$ & $\begin{array}{c}5.5 \\
\pm 2.4^{\mathrm{b}}\end{array}$ & $\begin{array}{c}1.9 \\
\pm 1.8\end{array}$ & $\begin{array}{r}4.6 \\
\pm 0.7^{\mathrm{a}}\end{array}$ & $4.0 \pm 1.1^{b}$ & $0.6 \pm 0.6$ & $3.1 \pm 0.9^{a}$ & $2.6 \pm 1.2^{\mathrm{b}}$ & $0.5 \pm 0.7$ & $2.7 \pm 1.6$ & $2.3 \pm 1.8$ & $0.5 \pm 1.0$ \\
\hline & $\begin{array}{c}\text { Mean } \\
\text { Severity }\end{array}$ & $\begin{array}{l}23.7 \\
\pm 9.7^{\mathrm{a}}\end{array}$ & $\begin{array}{c}12.4 \\
\pm 10.1^{\mathrm{b}}\end{array}$ & $\begin{array}{l}11.3 \\
\pm 5.3\end{array}$ & $\begin{array}{c}22.7 \\
\pm 9.6^{\mathrm{a}}\end{array}$ & $9.6 \pm 6.8^{b}$ & $10.1 \pm 4.9$ & $\begin{array}{c}10.5 \\
\pm 4.8^{\mathrm{a}}\end{array}$ & $5.3 \pm 4.2^{b}$ & $5.1 \pm 2.9$ & $9.3 \pm 7.5^{\mathrm{a}}$ & $\begin{array}{c}4.4 \\
\pm 5.3^{\mathrm{b}}\end{array}$ & $4.9 \pm 4.0$ \\
\hline \multirow[t]{2}{*}{ Male } & $\begin{array}{l}\text { Mean } \\
\text { Score }\end{array}$ & $7.9 \pm 1.4$ & $5.7 \pm 1.7$ & $\begin{array}{c}2.1 \\
\pm 1.8\end{array}$ & $4.9 \pm 0.4$ & $3.9 \pm 1.1$ & $1.0 \pm 1.2$ & $3.6 \pm 0.5$ & $2.9 \pm 1.1$ & $0.7 \pm 1.1$ & $2.7 \pm 1.3$ & $2.6 \pm 1.4$ & $0.1 \pm 0.4$ \\
\hline & $\begin{array}{c}\text { Mean } \\
\text { Severity }\end{array}$ & $\begin{array}{l}22.7 \\
\pm 5.9\end{array}$ & $9.6 \pm 5.3$ & $\begin{array}{l}13.1 \\
\pm 5.4\end{array}$ & $\begin{array}{c}15.9 \\
\pm 4.6^{\mathrm{a}}\end{array}$ & $7.1 \pm 4.2^{b}$ & $8.7 \pm 3.4$ & $9.1 \pm 3.5^{\mathrm{a}}$ & $4.6 \pm 2.5^{b}$ & $4.6 \pm 2.6$ & $7.7 \pm 5.6^{a}$ & $\begin{array}{c}3.7 \\
\pm 2.7^{\mathrm{b}}\end{array}$ & $4.0 \pm 3.2$ \\
\hline \multirow[t]{2}{*}{ Total } & $\begin{array}{l}\text { Mean } \\
\text { Score }\end{array}$ & $7.6 \pm 1.5^{\mathrm{a}}$ & $\begin{array}{c}5.6 \\
\pm 2.2^{\mathrm{b}}\end{array}$ & $\begin{array}{c}2.0 \\
\pm 1.7\end{array}$ & $\begin{array}{c}4.7 \\
\pm 0.7^{\mathrm{a}}\end{array}$ & $4.0 \pm 1.1^{\mathrm{b}}$ & $0.7 \pm 0.8$ & $3.3 \pm 0.8^{\mathrm{a}}$ & $2.7 \pm 1.2^{\mathrm{b}}$ & $0.6 \pm 0.9$ & $2.7 \pm 1.5$ & $2.4 \pm 1.6$ & $0.4 \pm 0.9$ \\
\hline & $\begin{array}{c}\text { Mean } \\
\text { Severity }\end{array}$ & $\begin{array}{l}23.4 \\
\pm 8.5^{\mathrm{a}}\end{array}$ & $\begin{array}{c}11.5 \\
\pm 8.7^{\mathrm{b}}\end{array}$ & $\begin{array}{l}11.9 \\
\pm 5.3\end{array}$ & $\begin{array}{c}20.5 \\
\pm 8.8^{\mathrm{a}}\end{array}$ & $8.8 \pm 6.1^{b}$ & $9.6 \pm 4.4$ & $\begin{array}{c}10.0 \\
\pm 4.4^{\mathrm{a}}\end{array}$ & $5.1 \pm 3.7^{b}$ & $5.0 \pm 2.7$ & $8.8 \pm 6.8^{a}$ & $\begin{array}{c}4.2 \\
\pm 4.5^{\mathrm{b}}\end{array}$ & $4.6 \pm 3.7$ \\
\hline \multicolumn{14}{|c|}{ No Head-Neck Cooling } \\
\hline \multirow[t]{2}{*}{ Female } & $\begin{array}{l}\text { Mean } \\
\text { Score }\end{array}$ & $7.4 \pm 2.2$ & $7.6 \pm 2.2$ & $\begin{array}{l}-0.1 \\
\pm 0.4\end{array}$ & $4.9 \pm 0.4$ & $4.9 \pm 0.4$ & $0.0 \pm 0.0$ & $3.6 \pm 0.5$ & $3.6 \pm 0.5$ & $0.0 \pm 0.0$ & $3.9 \pm 0.4$ & $3.9 \pm 0.4$ & $0.0 \pm 0.0$ \\
\hline & $\begin{array}{c}\text { Mean } \\
\text { Severity }\end{array}$ & $\begin{array}{l}20.1 \\
\pm 9.1\end{array}$ & $\begin{array}{l}20.1 \\
\pm 9.2\end{array}$ & $\begin{array}{c}0.0 \\
\pm 0.6\end{array}$ & $\begin{array}{l}18.7 \\
\pm 7.0\end{array}$ & $18.0 \pm 6.5$ & $0.7 \pm 1.5$ & $\begin{array}{l}12.6 \\
\pm 5.2\end{array}$ & $\begin{array}{l}12.3 \\
\pm 5.0\end{array}$ & $0.3 \pm 1.0$ & $\begin{array}{l}14.9 \\
\pm 6.0\end{array}$ & $\begin{array}{l}14.1 \\
\pm 5.4\end{array}$ & $0.7 \pm 1.3$ \\
\hline \multirow[t]{2}{*}{ Male } & $\begin{array}{l}\text { Mean } \\
\text { Score }\end{array}$ & $8.0 \pm 1.7$ & $8.0 \pm 1.7$ & $\begin{array}{c}0.0 \\
\pm 0.0\end{array}$ & $4.7 \pm 0.8$ & $4.7 \pm 0.8$ & $0.0 \pm 0.0$ & $3.5 \pm 0.8$ & $3.5 \pm 0.8$ & $0.0 \pm 0.0$ & $3.7 \pm 0.5$ & $3.5 \pm 0.6$ & $0.2 \pm 0.4$ \\
\hline & $\begin{array}{c}\text { Mean } \\
\text { Severity }\end{array}$ & $\begin{array}{l}22.3 \\
\pm 8.4\end{array}$ & $\begin{array}{l}23.0 \\
\pm 8.3\end{array}$ & $\begin{array}{l}-0.7 \\
\pm 1.2\end{array}$ & $\begin{array}{l}16.8 \\
\pm 4.1\end{array}$ & $16.8 \pm 4.1$ & $0.0 \pm 0.0$ & $\begin{array}{l}12.0 \\
\pm 3.0\end{array}$ & $\begin{array}{l}12.2 \\
\pm 2.9\end{array}$ & $\begin{array}{l}-0.2 \\
\pm 0.4\end{array}$ & $\begin{array}{l}12.2 \\
\pm 4.8\end{array}$ & $\begin{array}{l}12.0 \\
\pm 5.0\end{array}$ & $\begin{array}{l}0.27 \\
\pm 0.4\end{array}$ \\
\hline \multirow[t]{2}{*}{ Total } & $\begin{array}{l}\text { Mean } \\
\text { Score }\end{array}$ & $7.7 \pm 1.9$ & $7.8 \pm 1.9$ & $\begin{array}{l}-0.1 \\
\pm 0.3\end{array}$ & $4.8 \pm 0.6$ & $4.8 \pm 0.6$ & $0.0 \pm 0.0$ & $3.5 \pm 0.7$ & $3.5 \pm 0.7$ & $0.0 \pm 0.0$ & $3.8 \pm 0.4$ & $3.7 \pm 0.5$ & $0.1 \pm 0.3$ \\
\hline & $\begin{array}{c}\text { Mean } \\
\text { Severity }\end{array}$ & $\begin{array}{l}21.2 \\
\pm 8.5\end{array}$ & $\begin{array}{l}21.5 \\
\pm 8.6\end{array}$ & $\begin{array}{l}-0.3 \\
\pm 1.0\end{array}$ & $\begin{array}{l}17.9 \\
\pm 5.7\end{array}$ & $17.5 \pm 5.3$ & $0.4 \pm 1.1$ & $\begin{array}{l}12.3 \\
\pm 4.2\end{array}$ & $\begin{array}{l}12.2 \\
\pm 4.0\end{array}$ & $0.1 \pm 0.8$ & $\begin{array}{l}13.6 \\
\pm 5.5\end{array}$ & $\begin{array}{l}13.2 \\
\pm 5.1\end{array}$ & $0.5 \pm 1.0$ \\
\hline
\end{tabular}

$\mathrm{SD}=$ Standard Deviation; Significant difference $(\mathrm{p}<0.05)$ than $(\mathrm{a})=$ Post; $(\mathrm{b})=$ Pre .

\section{Discussion}

There is currently no recognized treatment for mTBI, and the management of mTBI continues to present significant challenges in both sporting and clinical settings, with rest and guided return to activity being the main stay of a brain injury management plan. Therefore, this study investigated the use of a selective headneck cooling device (Catalyst ${ }^{\mathrm{TM}}$ Cryohelmet) for the therapeutic management of symptoms associated with SOBI. The key finding observed and most pronounced was an immediate reduction in post-injury event clinical symptoms in both male and female players who wore the head-neck cooling device. Whilst players who did not elect to use the head-neck cooling device, demonstrated no change in symptom scores measured via the CSS.

Interestingly, females recorded a similar symptom score in all the symptom scale clusters when compared with males for both the selective and no head-neck cooling groups. However, they did record a higher severity in most, but not all of the symptom clusters which was similar to previous studies [45-47]. A study 
of high-school and college soccer players identified that females reported a greater number of symptoms than males and this was similar when reviewed by symptom clusters [46]. In a systematic review and meta-analysis [45] females had a greater number of concussion symptoms when compared with males. Interestingly the pre-monarchal females and menopausal women did not demonstrate meaningful differences in symptomatic presentation when compared with males, but differences were observed in women post-monarchal [45]. Gonadal hormone levels may account for these differences [48]. The females enrolled in this study were not asked to identify if they were pre- or post-monarchal and this may be something to consider in future research.

If post-monarchal status has any influence on the symptomatic presentation, then this may be a factor in the effects of the selective head-neck cooling on the reduction of symptoms as reported in this study. The findings from this study suggest that short duration, active head-neck cooling immediately after a SOBI may aid in acute phase recovery. The concept is biologically plausible given that temperature is an established regulator of neuronal functionality. Hyperthermia can impair cerebral autoregulation and that directed hypothermia can restore CBF and encourage cerebral heat clearance $[22,49]$. Our findings support those from Walter et al.,(2018) who reported that immediately following administration of 30 minutes of selective head-neck cooling, there was a marked improvement in $\mathrm{CBF}$ and the vast majority of mTBI participants reported a partial resolution of symptoms, predominantly physical symptoms including headache and dizziness.

In the present study, the average wear time was approximately 20 minutes which was shorter than a previous study [24] that used 60 minutes to achieve an average of $1.8^{\circ} \mathrm{C}$ (range 0.9 to $2.4^{\circ} \mathrm{C}$ ) decrease in brain temperature. The appropriate dosing and duration of selective head-neck cooling needs to be studied further. Evidence of the effect of longer duration hypothermia ( $>24$ hours) on outcomes in patients with traumatic and severe brain injury, has been found to be inconclusive [50,51]. With this form of prolonged cooling there was an increased risk for pneumonia [51]. It is currently unclear if there are any side effects from short term headneck cooling, although head surface cooling can increase systolic and diastolic blood pressures [32]. In the present study, there were no adverse effects reported. However, studies are warranted to further explore the potential adverse effects and safety of athletes within these circumstances.

A study used a head-neck cooling device for 60 minutes to achieve a brain temperature reduction of $1.84^{\circ} \mathrm{C}$ in patients with severe brain injury [24]. In a more recent study on healthy volunteers, [29] there was a noticeable decrease $\left(37.01 \pm 0.34^{\circ} \mathrm{C}\right.$ vs. $36.86 \pm 0.31^{\circ} \mathrm{C}$ ) in tympanic temperature at 30 minutes but not at any further time points. This was also the same time utilised for healthy and concussed college-aged athletes [28]. As most matches are of 60 to 80 minutes duration this time frame was identified as being achievable and the minimum and maximum duration of selective head-neck cooling for the purpose of the research was 15 and 30 minutes, respectively. Although not formally reported, SOBI players described an improvement in feelings of wellbeing when using the Cryohelmet, and that their head instantly felt calmer and cooler similar to a previous study [28]. It has been suggested [28] that as a result of selective head-neck cooling, compromised neurovascular coupling may be temporary restored to match the CBF with surges in the metabolic demands of the brain. The paradoxical increase in CBF may have been adequate enough to meet the metabolic demands of the brain occurring and that the clinical symptoms of concussion are alleviated, albeit temporarily [28]. However, given the effects of selective head-neck cooling with symptom reduction there is the possibility that more frequent or longer duration cooling may be beneficial. Nevertheless, it is important to acknowledge that the improvement or resolution of the clinical symptoms of SOBI does not sufficiently reflect healing of a brain injury, [52-55] and therefore return-to-play decisions should not be made on the basis of symptoms following sideline, selective head-cooling.

\section{Limitations}

There were several limitations to this study that must be acknowledged. First, although there were noticeable differences in clinical symptom scores and severity for the cooling cohort compared with the non-cooling cohort, the limited sample size may not have been enough to enable the identification of a meaningful difference in the results. Second, it was not clear if improvements in symptoms after cooling were long lasting. No further follow up was undertaken with the cooling to identify what effects selective cooling may have on clinical symptoms and the severity of SOBI over the longer term or if the post injury improvement in symptoms was temporary or sustained. A previous study has reported only temporary beneficial effects from the selective head-neck cooling and symptoms returned shortly after the intervention was stopped, although this was also attributed to other factors such as noise from the MRI system [28]. Third, no temperature evaluation was undertaken on the sideline given that the games were primarily played outdoors during the New Zealand winter season. Lastly, no female collision-sport participants selected to not wear the Cryohelmet for the sideline post-event evaluation. Although some male participants did select a period of rest post initial assessment and there were some noticeable differences, this may have been different for female collision-sports participants given females have been known to report more concussion symptoms and take longer to recover $[45,56,57]$. Despite the aforementioned limitations, this study identified an observed and pronounced immediate reduction in post-brain injury event clinical symptoms for both male and female players with selective head-neck cooling. Collectively, future research should look to investigate the specifics surrounding, duration, frequency, time since injury (acute, sub-acute, chronic phases) as well as underlying mechanisms that contribute to reduction in such symptoms. 


\section{Conclusion}

Short-term, selective head-neck cooling offers potential as an effective therapeutic strategy for the acute management of the symptoms of SOBI. Further studies are required to test the underlying mechanisms, dose and duration, sustainability of improvements, effects on biomarkers, and the feasibility and safety of head-cooling.

\section{Competing Interests}

The authors declare that there are no competing interests associated with the research contained within this manuscript.

\section{Funding}

No source of funding was utilized in the conducting of this study.

\section{Contributor Statement}

The corresponding author attests that all listed authors meet authorship criteria and that no others meeting the criteria have been omitted. According to the definition given by the International Committee of Medical Journal Editors (ICMJE), the authors listed above qualify for authorship based on making one or more of the substantial contributions to the intellectual content of:

a) Conception and design [DK; PH]; and/or,

b) Acquisition of data [DK]; and/or

c) Analysis and interpretation of data [DK, PH, TC]; and/or

d) Participated in drafting of the manuscript [DK, PH, KH, TC, CC]; and/or

e) Critical revision of the manuscript for important intellectual content [DK, $\mathrm{PH}, \mathrm{KH}, \mathrm{TC}, \mathrm{CC}]$.

\section{References}

1. Wang H, Wang B, Jackson K, Miller CM, Hasadsri L, et al. (2015) A novel head-neck cooling device for concussion injury in contact sports. Transl Neurosci 6(1): 20-31.

2. Wang H, Kim M, Normoyle K, Llano D (2016) Thermal regulation of the brain - An anatomical and physiological review for clinical neuroscientists Front Neurosci 9(528).

3. Howarth C, Gleeson P, Attwell D (2012) Updated energy budgets for neural computation in the neocortex and cerebellum. J Cereb Blood Flow Metab 32(7): 1222-1232.

4. Godek SF, Bartolozzi AR, Godek JJ (2005) Sweat rate and fluid turnover in American football players compared with runners in a hot and humid environment. Br J Sports Med 39(4): 205-211.

5. Titus DJ, Furones C, Atkins CM, Dietrich WD (2015) Emergence of cognitive deficits after mild traumatic brain injury due to hyperthermia. Exp Neurol 263: 254-262.

6. Nybo L, Møller K, Volianitis S, Nielsen B, Secher NH (2002) Effects of hyperthermia on cerebral blood flow and metabolism during prolonged exercise in humans. J Appl Physiol 93(1): 58-64.

7. Nybo L, Nielsen B (2001) Middle cerebral artery blood velocity is reduced with hyperthermia during prolonged exercise in humans. J Physiol 534(Pt 1): 279-286.

8. White MD, Cabanac M (1996) Exercise hyperpnea and hyperthermia in humans. J Appl Physiol. 81(3): 1249-1254.
9. Hayward JN, Baker MA (1969) A comparative study of the role of the cerebral arterial blood in the regulation of brain temperature in five mammals. Brain Res 16(2): 417-440.

10. Rasmussen P, Stie H, Nielsen B, Nybo L (2006) Enhanced cerebral CO2 reactivity during strenuous exercise in man. Eu J Appl Physiol 96(3): 299-304.

11. Williamson JW, Mc Coll R, Mathews D, Ginsburg M, Mitchell JH (1999) Activation of the insular cortex is affected by the intensity of exercise. J App Physiol 87(3): 1213-1219.

12. Kochanek PM, Jackson TC (2015) It might be time to let cooler heads prevail after mild traumatic brain injury or concussion. Exp Neurol 267: 13-17.

13. Madsen PL, Sperling BK, Warming T, Schmidt JF, Secher NH, et al. (1993) Middle cerebral artery blood velocity and cerebral blood flow and 02 uptake during dynamic exercise. J App Physiol74(1): 245-250.

14. Nybo L, Secher NH, Nielsen B (2002) Inadequate heat release from the human brain during prolonged exercise with hyperthermia. J Physiol 545(Pt 2): 697-704.

15. Sakurai A, Atkins CM, Alonso OF, Bramlett HM, Dietrich WD (2012) Mild hyperthermia worsens the neuropathological damage associated with mild traumatic brain injury in rats. J Neurotrauma 29(2): 313-321.

16. Giza C, Hovda D (2014) The new neurometabolic cascade of concussion. Neurosurg 75(S4): S24-S33.

17. Bailes J, Petraglia A, Omalu B, Nauman E, Talavage (2013) Role of subconcussion in repetitive mild traumatic brain injury. J Neurosurg 119(5): 1235-1245.

18. Dietrich W, Bramlett $H$ (2010) The evidence for hypothermia as a neuroprotectant in traumatic brain injury. Neurotherapeutics 7(1): 4350.

19. Hajat C, Hajat S, Sharma P (2000) Effects of poststroke pyrexia on stroke outcome. Stroke 31(2): 410-414.

20. Li J, Jiang Jy (2012) Chinese head trauma data bank: Effect of hyperthermia on the outcome of acute head trauma patients. J Neurotrauma 29(1): 96100 .

21. Erecinska M, Thoresen M, Silver IA (2003) Effects of hypothermia on energy metabolism in mammalian central nervous system. J Cereb Blood Flow Metab 23(5): 513-530.

22. Yenari M, Wijman C, Steinberg G (2004) Effects of hypothermia on cerebral metabolism, blood flow and autoregulation. In: Mayer S, Sessler D (Eds.). Therapeutic Hypothermia. ( $1^{\text {st }}$ Edn), CRC Press Taylor \& Francis, New York, USA, pp. 141-178.

23. Dietrich WD, Atkins CM, Bramlett HM (2009)Protection in animal models of brain and spinal cord injury with mild to moderate hypothermia. J Neurotrauma 26(3): 301-312.

24. Wang H, Olivero W, Lanzino G, Elkins W, Rose J, et al. (2004) Rapid and selective cerebral hypothermia achieved using a cooling helmet. J Neurosurg 100(2): 272-277.

25. Gluckman P, Wyatt J, Azzopardi D, Ballard R, Edwards A, et al. (2005) Selective head cooling with mild systemic hypothermia after neonatal encephalopathy: multicentre randomised trial. Lancet 365(9460): 663670 .

26. Bernard SA, Buist M (2003) Induced hypothermia in critical care medicine: A review. Crit Care Med. 31(7): 2041-2051.

27. Bernard SA, Gray TW, Buist MD, Jones BM, Silvester W, et al. (2002) Treatment of comatose survivors of out-of-hospital cardiac arrest with induced hypothermia. N Engl J Med 346(8): 557-563.

28. Walter A, Finelli K, Bai X, Johnson B, Neuberger T, et al. (2018) Neurobiological effect of selective brain cooling after concussive injury. Brain Imaging Behav 12(3): 891-900.

29. Jackson K, Rubin R, Van Hoeck N, Hauert T, Lana V, et al. (2015) The effect of selective head-neck cooling on physiological and cognitive functions in healthy volunteers. Transl Neurosci 6(1): 131-138. 
30. Kallmünzer B, Beck A, Schwab S, Kollmar R (2011) Local head and neck cooling leads to hypothermia in healthy volunteers. Cerebrovas Dis 32(3): 207-210.

31. Miyauchi T, Wei EP, Povlishock JT (2014) Evidence for the therapeutic efficacy of either mild hypothermia or oxygen radical scavengers after repetitive mild traumatic brain injury. J Neurotrauma. 31(8): 773-781.

32. Koehn J, Kollmar R, Cimpianu CL, Kallmünzer B, Moeller S, et al. (2012) Head and neck cooling decreases tympanic and skin temperature, but significantly increases blood pressure. Stroke 43(8): 2142-2148.

33. Lovell M, Iverson G, Collins M, Podell K, Johnston K, et al. (2006) Measurement of symptoms following sports-related concussion: reliability and normative data for the post-concussion scale. App Neuropsychol 13(3): 166-174.

34. Hume P, KIng D, Mc Geown J, Theadom A (2019) Sports-related concussion, mild traumatic brain injury or sport-originated brain injury (SOBI): A more useful term. NZ J Sports Med 45(2): 64-67.

35. King D, Hume PA, Hardaker N, Cummins C, Gissane C, et al. (2019) Sports-related injuries in New Zealand: National Insurance (Accident Compensation Corporation) claims for five sporting codes from 2012 to 2016. Br J Sports Med 53(16): 1026-1033

36. King D, Hume PA, Hardaker N, Pearce A, Cummins C, et al. (2019) Traumatic brain injuries in New Zealand: National Insurance (Accident Compensation Corporation) claims from 2012 to 2016. J Neurol Sci. 399(2019): 61-68.

37. Notebaert A, Guskiewicz K (2005) Current trends in athletic training practice for concussion assessment and management. J Athl Train 40(4): 320-325.

38. Broglio S, Macciocchi S, Ferrara M (2007) Sensitivity of the concussion assessment battery. Neurosurgery 60(6): 1050-1058.

39. Pardini D, Stump J, Lovell M, Collins M, Moritz K, et al. (2004) The postconcussion symptom scale (PCSS): A factor analysis. Br J Sports Med 38(5): 661.

40. Merritt V, Arnett P (2014) Premorbid predictors of postconcussion symptoms in collegiate athletes. J Clin Exp Neuropsychol 36(10): 10981111

41. Merritt V, Meyer J, Arnett P (2015) A novel approach to classifying postconcussion symptoms: The application of a new framework to the Post-Concussion Symptom Scale. J Clin Exp Neuropsychol 37(7): 764775.

42. Derogatis L (1994) SCL-90-R administration, scoring, and procedures manual. ( $3^{\text {rd }}$ edn.), MN: National Computer Systems, Minneapolis,USA.

43. Twellaar M, Verstappen F, Huson A (1996) Is prevention of sports injuries a realistic goal? A four-year prospective investigation of sports injuries among physical education students. Am J Sports Med 24(4): 528-534.

\section{ISSN: 2574-1241}

DOI: 10.26717/BJSTR.2019.21.003639

Doug King. Biomed J Sci \& Tech Res

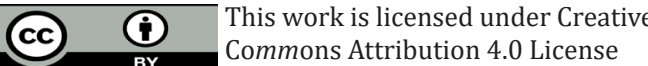

Submission Link: https://biomedres.us/submit-manuscript.php
44. Hopkins W, Marshall S, Batterham A, Hanin J (2009) Progressive statistics for studies in sports medicine and exercise science. Med Sci Sports Exerc 41(1) : 3-12.

45. Brown DA, Elsass JA, Miller AJ, Reed LE, Reneker JC (2015) Differences in symptom reporting between males and females at baseline and after a sports-related concussion: A systematic review and meta-analysis. Sports Med 45(7): 1027-1040.

46. Covassin T, Elbin R, Bleecker A, Lipchik A, Kontos A (2013) Are there differences in neurocognitive function and symptoms between male and female soccer players after concussions? Am J Sports Med. 41(12): 2890-2895.

47. Merritt V, Padgett C, Jak A (2019) A systematic review of sex differences in concussion outcome: What do we know? Clin Neuropsychol 33(6):1016-1043.

48. Bazarian JJ, Blyth B, Mookerjee S, He H, Mc Dermott MP (2010) Sex differences in outcome after mild traumatic brain injury. J Neurotrauma 27(3): 527-539.

49. Williams GR, Spencer FC (1958) The clinical use of hypothermia following cardiac arrest. Ann Surg. 148(3): 462-468

50. Madden LK, De Von HA (2015) A systematic review of the effects of body temperature on outcome after adult traumatic brain injury. J Neurosci Nurs 47(4): 190-203.

51. Lewis SR, Evans DJW, Butler AR, Schofield Robinson OJ, Alderson P (2017) Hypothermia for traumatic brain injury. Cochrane Database Syst Rev 21(9).

52. Johnson B, Zhang K, Gay M, Horovitz S, Hallett M, et al. (2012) Alteration of brain default network in subacute phase of injury in concussed individuals: Resting-state fMRI study. Neuroimage 59(1): 511-518.

53. Slobounov SM, Zhang K, Pennell D, Ray W, Johnson B, et al. (2010) Functional abnormalities in normally appearing athletes following mild traumatic brain injury: A functional MRI study. Exp Brain Res 202(2): 341-354.

54. Talavage T, Nauman E, Breedlove E, Yoruk U, Dye A, et al. (2014) Functionally-detected cognitive impairment in high school football players without clinically-diagnosed concussion. J Neurotrauma 31(4): 327-338.

55. Zhu DC, Covassin T, Nogle S, Doyle S, Russell D, et al. (2015) A potential biomarker in sports-related concussion: Brain functional connectivity alteration of the default-mode network measured with longitudinal resting-state fMRI over thirty days. J Neurotrauma 32(5): 327-341.

56. Mollayeva T, El Khechen Richandi G, Colantonio A (2018) Sex \& gender considerations in concussion research. Concussion 3(1): CNC51.

57. Mollayeva T, Mollayeva S, Colantonio A (2018) Traumatic brain injury: Sex, gender and intersecting vulnerabilities. Nat Rev Neurol 14(12): 711-722.

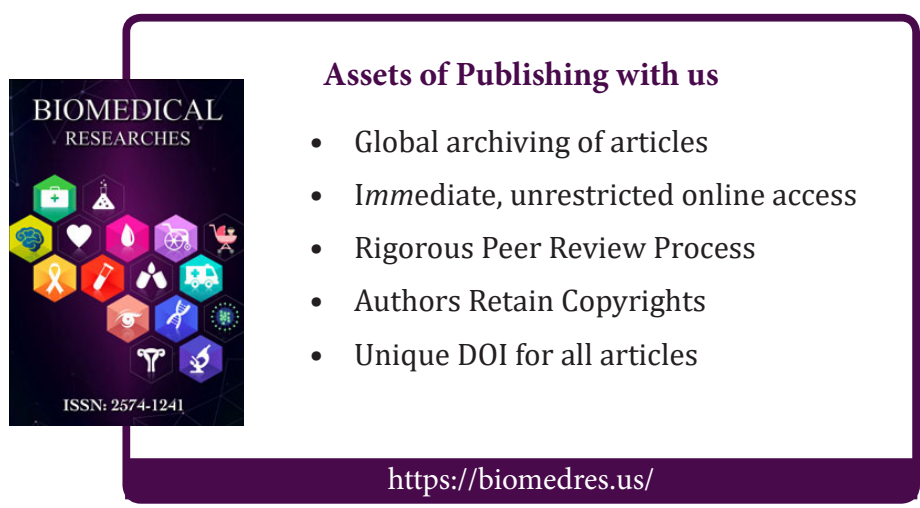

Copyright@ Doug King | Biomed J Sci \& Tech Res | BJSTR. MS.ID.003639. 